\title{
High expression of ZEB1 correlates with liver metastasis and poor prognosis in colorectal cancer
}

\author{
GUANG-JUN ZHANG, TONG ZHOU, HONG-PENG TIAN, ZUO-LIANG LIU and SHU-SEN XIA \\ The First Department of General Surgery, The Affiliated Hospital of North Sichuan Medical College, \\ Nanchong, Sichuan 637000, P.R. China
}

Received September 6, 2012; Accepted November 7, 2012

DOI: $10.3892 / \mathrm{ol} .2012 .1026$

\begin{abstract}
Zinc finger E-box binding homeobox 1 (ZEB1) has been shown to promote invasion and metastasis in several types of human cancer and to have a prognostic role in certain cancers. However, the clinical significance of ZEB1 in colorectal cancer (CRC) has not been sufficiently investigated. This study aimed to address this issue. In this study, we compared the expression of ZEB1 between CRC tissues and normal adjacent mucosa using quantitative real-time RT-PCR. The association of ZEB1 expression with clinicopathological characteristics was analyzed by appropriate statistical analyses. Kaplan-Meier analysis and Cox proportional hazards regression models were used to investigate the association of ZEB1 expression with survival of patients. The results showed that the relative expression levels of ZEB1 were significantly higher in CRC tissues compared to the normal adjacent mucosa and higher expression of ZEB1 correlated with liver metastasis. Kaplan-Meier analysis indicated that patients with high ZEB1 had a poor overall survival. Moreover, the multivariate analysis showed that high expression of ZEB1 was an independent predictor of overall survival. Our data indicate the potential of ZEB1 as a novel prognostic biomarker for CRC.
\end{abstract}

\section{Introduction}

Colorectal cancer (CRC) is the third most common cancer worldwide with an estimated one million new cases and half a million mortalities every year (1). A significant number of patients with colorectal carcinoma who undergo apparently curative surgery develop local recurrence or distant metastasis, leading to shorter survival (2). For this reason, the identification of factors that accurately predict prognosis in $\mathrm{CRC}$ is

Correspondence to: Dr Tong Zhou, The First Department of General Surgery, The Affiliated Hospital of North Sichuan Medical College, Nanchong, Sichuan 637000, P.R. China

E-mail: zhoutong0088@163.com

Key words: zinc finger e-box binding homeobox 1, colorectal cancer, prognostic factor urgently required. A deeper insight into the carcinogenesis and the factors correlated with the aggressiveness of CRC may be necessary for this requirement.

Several previous studies have revealed that epithelialmesenchymal transition (EMT) plays a crucial role in the progression and aggressiveness of CRC $(3,4)$. Several genes, called EMT-inducing or master EMT genes, are essential to the EMT process $(5,6)$. Zinc finger E-box binding homeobox 1 (ZEB1) encodes a transcription factor that plays a key role in cancer progression by regulating EMT in breast, prostate, ovarian and colorectal cancer (7-11).

A previous study showed that ZEB1 was overexpressed in various $C R C$ cell lines and crucial for the metastasis of CRC cells (12). Recently, ZEB1 was also found to be a crucial EMT inducer in human CRC and suppresses the expression of basement membrane components (13). Notably, EMT-linked loss of basement membranes indicates metastasis and poor survival in CRC $(7,14)$.

Although a growing number of studies have demonstrated the function of ZEB1 in experimental systems, no reports have shown any clinical significance associated with ZEB1 expression in CRC. The aim of this study was therefore to clarify the clinical significance of ZEB1 expression in CRC.

\section{Materials and methods}

Patients and tissue samples. After obtaining adequate informed consent, surgical specimens of cancer tissue and adjacent normal mucosa were obtained from 92 patients with primary CRC who underwent surgery without preoperative treatment at the First Department of General Surgery, the Affiliated Hospital of North Sichuan Medical College, China, between January 2005 and May 2008. All tissue samples were immediately frozen in liquid nitrogen and stored at $-80^{\circ} \mathrm{C}$ until the extraction of RNA. Clinicopathological information, including age, gender, tumor size, histological type, depth of invasion, lymph node metastasis, location, lymphatic invasion and liver metastasis, was available for all patients. The study was approved by the medical ethics committee of North Sichuan Medical College.

Quantitative real-time reverse transcription-PCR. Total RNA was extracted from tumor tissue and adjacent normal mucosa by homogenizing tissue in TRIzol reagent (Invitrogen, Carlsbad, 
CA, USA), according to the manufacturer's instructions. cDNA was synthesized using an iScript cDNA Synthesis kit (Bio-Rad Laboratories, Hercules, CA, USA). Quantitative real-time PCR was performed with an iQ SYBR-Green Supermix (Bio-Rad). All reactions were run in triplicate on the iCycler IQ multicolor Detection System (Bio-Rad). The amplification profile was denatured at $95^{\circ} \mathrm{C}$ for $10 \mathrm{~min}$, followed by 50 cycles of denaturation at $95^{\circ} \mathrm{C}$ for $30 \mathrm{sec}$, annealing at $60^{\circ} \mathrm{C}$ for $30 \mathrm{sec}$ and extension at $72^{\circ} \mathrm{C}$ for $1 \mathrm{~min}$. The primers for ZEB1 (226 bp) were 5'-AGCAGTGAAAGAGAAGGGAATGC-3' (sense) and 5'-GGTCCTCTTCAGGTGCCTCAG-3' (antisense). The primers for $\beta$-actin (171 bp) were 5'-AGT TGCGTTACACCCTTTCTTGAC-3' (sense) and 5'-GCTCGC TCCAACCGACTGC-3' (antisense). The comparative cycle threshold (CT) method was applied to quantify the expression levels of miRNAs. The relative amount of ZEB1 was calculated using the equation $2^{-\Delta \mathrm{C}}{ }_{\mathrm{T}}$ where $\Delta \mathrm{C}_{\mathrm{T}}=\left(\mathrm{C}_{\mathrm{T} \text { ZEB1 }}-\mathrm{C}_{\mathrm{T} \beta \text {-actin }}\right)$.

Statistical analysis. The gene expression levels in CRC were compared with those in normal adjacent mucosa with the use of the Wilcoxon test. The correlations between the gene expression levels and potential explanatory variables, including age, gender, tumor size, histological type, depth of invasion, lymph node metastasis, location, lymphatic invasion and liver metastasis, were evaluated with the Chi-square test. The postoperative survival rate was analyzed with the KaplanMeier method, and differences in survival rates were assessed with the log-rank test. A Cox proportional hazards model was used for multivariate analysis. All the statistical analyses were performed using SPSS 16.0 software (SPSS, Chicago, IL, USA). Two-sided P-values were calculated, and $\mathrm{P}<0.05$ was considered to indicate a statistically significant result.

\section{Results}

Comparison of ZEBI expression between CRC tissue and normal adjacent mucosa. By real-time quantitative RT-PCR, we found that ZEB1 expression levels were significantly higher in cancer tissues from patients with CRC $(0.843 \pm 0.693)$ than in the normal adjacent mucosa $(0.586 \pm 0.488 ; \mathrm{P}=0.003$; Fig. 1).

Correlation between ZEB1 gene expression and clinicopathological features of $C R C$. The expression levels of ZEB1 were categorized as low or high in relation to the median value. The ZEB1 expression level was not correlated with age, gender, tumor size, histological type, depth of invasion, tumor location, lymph node metastasis or lymphatic invasion. However, the ZEB1 expression level was correlated with liver metastasis $(\mathrm{P}=0.043$; Table I).

Correlation between ZEB1 gene expression levels and survival. Overall survival curves were plotted according to ZEB1 mRNA expression level by the Kaplan-Meier method. In the study group as a whole (92 patients), the overall survival rate was significantly lower in the patients with high ZEB1 mRNA expression than in those with low expression ( $\mathrm{P}=0.010$; Fig. 2).

Prognostic factors of CRC. Univariate analysis with Cox proportional hazards model identified seven prognostic factors: histological type, tumor size, depth of invasion, lymph
Table I. Correlation between ZEB1 expression and clinicopathological parameters in 92 colorectal cancer patients.

\begin{tabular}{|c|c|c|c|}
\hline \multirow[b]{2}{*}{ Variable } & \multicolumn{2}{|c|}{ ZEB1 expression } & \multirow[b]{2}{*}{ P-value } \\
\hline & $\begin{array}{c}\text { Low } \\
(n=46)\end{array}$ & $\begin{array}{l}\text { High } \\
(n=46)\end{array}$ & \\
\hline Age (years) & $62.8 \pm 14.6$ & $61.1 \pm 11.7$ & 0.519 \\
\hline Gender & & & 0.209 \\
\hline Male & 28 & 22 & \\
\hline Female & 18 & 24 & \\
\hline Tumor size (cm) & & & 0.288 \\
\hline$\leq 5$ & 30 & 25 & \\
\hline$>5$ & 16 & 21 & \\
\hline Histological type & & & 0.529 \\
\hline Well, moderate & 27 & 24 & \\
\hline Poor, mucinous & 19 & 22 & \\
\hline Depth of invasion & & & 0.674 \\
\hline $\mathrm{T} 1, \mathrm{~T} 2$ & 21 & 19 & \\
\hline $\mathrm{T} 3, \mathrm{~T} 4$ & 25 & 27 & \\
\hline Location & & & 0.669 \\
\hline Colon & 19 & 17 & \\
\hline Rectum & 27 & 29 & \\
\hline Lymph node metastasis & & & 0.058 \\
\hline Absent & 24 & 15 & \\
\hline Present & 22 & 31 & \\
\hline Lymph node invasion & & & 0.084 \\
\hline Absent & 33 & 25 & \\
\hline Present & 13 & 21 & \\
\hline Liver metastasis & & & $0.043^{\mathrm{a}}$ \\
\hline Absent & 40 & 32 & \\
\hline Present & 6 & 14 & \\
\hline
\end{tabular}

ZEB1, zinc finger E-box binding homeobox 1; n, number of patients; well, well-differentiated; moderate, moderately differentiated; poor, poorly differentiated.

node invasion, lymphatic invasion, liver metastasis and ZEB1 expression. The other clinicopathological features, such as age, gender and location, were not statistically significant prognostic factors (Table II). A multivariate analysis of the prognosis factors with a Cox proportional hazards model confirmed that high ZEB1 expression was a significant independent predictor of poor survival in CRC (Table III).

\section{Discussion}

Increasing evidence indicates that aberrant activation of EMT plays a key role in tumor cell invasion and metastasis. EMT allows the detachment of cells from each other and increases cell mobility, both of which are necessary for tumor cell dissemination. A hallmark for EMT is the loss of the cell adhesion molecule E-cadherin. Several transcription factors have been described as key inducers of EMT, including members 
Table II. Univariate analysis of clinicopathological factors for overall survival.

\begin{tabular}{lccc}
\hline Variable & Hazard & & \\
ratio & $95 \% \mathrm{CI}$ & P-value
\end{tabular}

Age (years)

$\begin{array}{llcll}\leq 65 & 40 & 1 & & \\ >65 & 52 & 1.455 & 0.701-3.019 & 0.314\end{array}$

Gender

Male

Female

$50 \quad 1$

Tumor size $(\mathrm{cm})$

$\begin{array}{ccccc}\leq 5 & 55 & 1 & & \\ >5 & 37 & 3.837 & 1.771-8.312 & 0.001^{\mathrm{a}}\end{array}$

Histological type

Well, moderate

Poor, mucinous

$51 \quad 1$

$41 \quad 2.401$

$1.141-5.052$

$0.021^{\mathrm{a}}$

Depth of invasion

T1, T2

T3, T4

$$
40 \quad 1
$$

$52 \quad 3.247$

1.383-7.625

$0.007^{\mathrm{a}}$

Location

\section{Colon}

Rectum

$36 \quad 1$

Lymph node

metastasis

$\begin{array}{ccccc}\text { Absent } & 39 & 1 & & \\ \text { Present } & 53 & 8.956 & 2.704-29.663 & <0.001^{\mathrm{a}}\end{array}$

Lymph node

invasion

$\begin{array}{llccc}\text { Absent } & 58 & 1 & & \\ \text { Present } & 34 & 3.820 & 1.797-8.121 & <0.001^{\mathrm{a}}\end{array}$

Liver metastasis

\begin{tabular}{ccccc} 
Absent & 72 & 1 & & \\
Present & 20 & 15.427 & $6.342-37.528$ & $<0.001$ \\
ZEB1 & & & & \\
Low & 46 & 1 & & \\
High & 46 & 2.646 & $1.226-5.710$ & $0.013^{\mathrm{a}}$ \\
\hline
\end{tabular}

$\mathrm{n}$, number of patients; CI, confidence interval; ZEB1, zinc finger E-box binding homeobox 1; well, well-differentiated; moderate, moderately differentiated; poor, poorly differentiated. ${ }^{\mathrm{a}} \mathrm{P}<0.05$.

of the Snail superfamily (Snail1 and Snail2), the basic helixloop-helix (bHLH) family (TCF3 and TWIST) and the two zinc-finger E-box-binding homeobox (ZEB) factors (ZEB1 and ZEB2) $(15,16)$.

Previous data indicate that ZEB1 has emerged as a key player in cancer progression $(8,12,17,18)$. Aberrant expression of ZEB1 in endometrial cancers, gastric cancer and hepatocellular carcinoma has been associated with aggressive disease, poor differentiation, development of metastases and poor clinical prognosis (19-21). However, ZEB1 expression in CRC tissues and its correlation with the clinical pathology of CRC are rarely reported.
Table III. Multivariate analysis of clinicopathological factors for overall survival.

\begin{tabular}{lccc}
\hline Variable & $\begin{array}{c}\text { Hazard } \\
\text { ratio }\end{array}$ & $95 \%$ CI & P-value \\
\hline $\begin{array}{l}\text { Tumor size } \\
(>5 \mathrm{~cm} / \leq 5 \mathrm{~cm})\end{array}$ & 1.779 & $0.736-5.044$ & 0.182 \\
$\begin{array}{l}\text { Histological type } \\
\text { (poor, muc/well, mod) }\end{array}$ & 1.852 & $0.841-4.079$ & 0.126 \\
$\begin{array}{l}\text { Depth of invasion } \\
\text { (T3, T4/T1, T2) }\end{array}$ & 1.548 & $0.620-3.864$ & 0.349 \\
$\begin{array}{l}\text { Lymph node metastasis } \\
\text { (present/absent) }\end{array}$ & 6.165 & $1.733-21.926$ & $0.005^{\mathrm{a}}$ \\
$\begin{array}{l}\text { Lymphatic invasion } \\
\text { (present/absent) }\end{array}$ & 2.001 & $0.788-5.080$ & 0.144 \\
$\begin{array}{l}\text { Liver metastasis } \\
\text { (present/absent) }\end{array}$ & 4.816 & $1.794-12.929$ & $0.002^{\mathrm{a}}$ \\
$\begin{array}{l}\text { ZEB1 (high/low) } \\
\text { (pEs }\end{array}$ & 2.237 & $1.008-4.968$ & $0.048^{\mathrm{a}}$ \\
\hline
\end{tabular}

CI, confidence interval; well, well-differentiated; mod, moderately differentiated; poor, poorly differentiated; muc, mucinous; ZEB1, zinc finger E-box binding homeobox 1. ${ }^{\mathrm{a}} \mathrm{P}<0.05$.

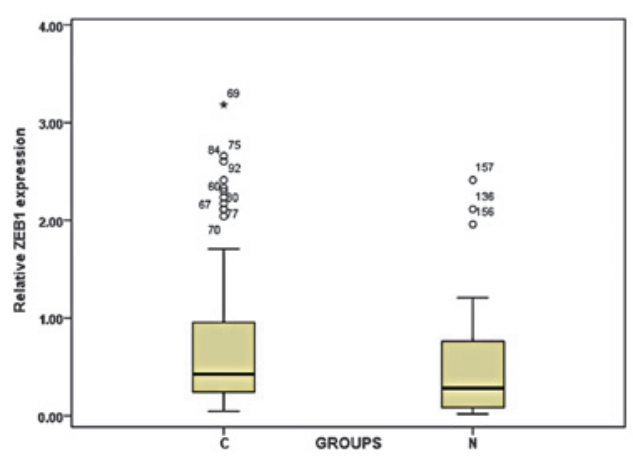

Figure 1. Comparison of zinc finger E-box binding homeobox 1 (ZEB1) expression levels between colorectal cancer tissue $(\mathrm{C})$ and adjacent normal mucosa $(\mathrm{N})$. P-value was calculated by the Wilcoxon test.

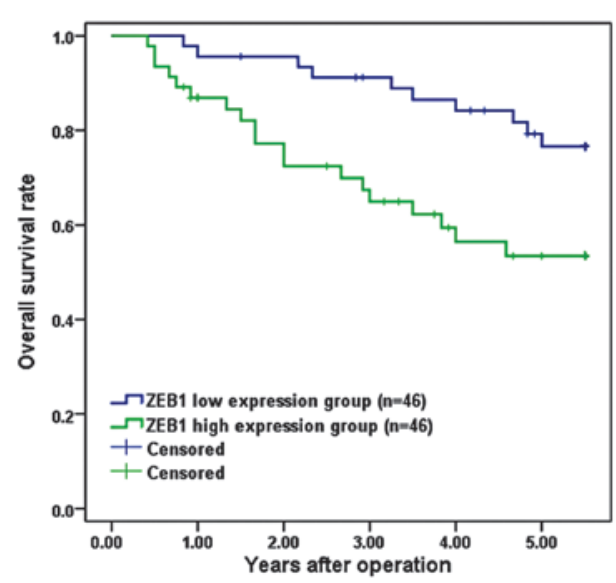

Figure 2. Kaplan-Meier survival curves of patients with colorectal cancer based on zinc finger E-box binding homeobox 1 (ZEB1) expression status. Patients in the high expression group (green line) had significantly poorer prognosis than those in the low expression group (blue line; $\mathrm{P}=0.010$, log-rank test). 
The overexpression of ZEB1 has been observed in prostate cancer, gastric cancer, osteosarcoma and hepatocellular carcinoma, suggesting an important role in tumorigenesis $(11,20-22)$. A previous study also showed that ZEB1 was overexpressed in various CRC cell lines (12). In this study, we confirmed that ZEB1 gene expression was higher in CRC tissue than in adjacent normal mucosa, consistent with the results of previous studies.

A previous study demonstrated that high ZEB1 expression in hepatocellular carcinoma was correlated with advanced TNM stage, tumor size, intrahepatic metastasis and vascular invasion (21). Okugawa et al reported that increased ZEB1 expression in gastric cancer was not clearly associated with histological type, tumor size, lymph node metastasis and hepatic metastasis, and elevated ZEB1 expression was shown to be significantly correlated with peritoneal dissemination (20). However, the correlation between ZEB1 expression level and clinicopathological behavior of CRC is unclear. In the present study, ZEB1 expression was shown to be associated only with liver metastases, suggesting that ZEB1 is involved in the carcinogenesis, development, progression and metastasis of CRC. The inconsistent results may be due to different roles of ZEB1 in different tumors.

More importantly, we proved that ZEB1 expression was significantly associated with overall survival of patients with CRC. In support of this, the Kaplan-Meier analysis of overall survival showed that patients whose tumors had higher ZEB1 expression tend to have a significantly worse overall survival, indicating that a high ZEB1 level is a marker of poor prognosis for patients with CRC. Moreover, Cox proportional hazards model showed that ZEB1 was a marker of poor overall survival independent of the known clinical prognostic indicators such as lymph node and liver metastases. Therefore, it could constitute a molecular prognostic marker for these patients, identifying those patients who are more likely to have a higher risk of mortality; thus, good candidates to receive more aggressive treatment.

The precise molecular mechanisms behind the altered expression of ZEB1 in CRC are unclear. To the best of our knowledge, this is the first study to describe the significance of ZEB1 to liver metastasis and prognosis of CRC patients. ZEB1, which is also known as TCF8 or $\triangle \mathrm{EF} 1$, is a member of the zinc finger family (22). It had been found that ZEB1 inhibits the expression of multiple genes in a variety of cell lines. In hematopoietic cells, ZEB1 negatively regulated the expression levelss of IL-2, CD4, the heavy chain of immunoglobulin $\mu$ and GATA-3 (23-25). In mesenchymal cells, ZEB1 inhibited the expression of the p73 gene (26). Recent studies found that ZEB1 also inhibited the expression of epithelial cadherin (E-cadherin), and thus plays an important role in the carcinogenesis, progression, invasion and metastasis of a variety of tumors $(27,28)$. Putzke et al reported that the overexpression of ZEB-1 in prostate cancer cells inhibited the expression of E-cadherin protein, thereby promoting the invasion and metastasis of prostate cancer cells (27). The overexpression of ZEB1 was also found to promote the invasion and metastasis of lung cancer cells (29). In addition, ZEB1 promotes the epithelialmesenchymal transformation of liver cancer cells (30). Taken together, these studies may explain why ZEB1 overexpression is associated with poor prognosis of CRC patients. Additional studies to investigate the molecular mechanisms of both the cause and effects of altered expression of ZEB1 in the development and/or progression of CRC are essential.

In conclusion, we have shown that ZEB1 expression was increased in clinical CRC specimens and associated with liver metastasis and poor prognosis. This study further demonstrated that ZEB1 was an independent prognostic factor of patients with CRC. These findings suggest that ZEB1 may be a potential diagnostic and therapeutic target in patients with CRC.

\section{Acknowledgements}

This study was in part supported by the Scientific Research Program of Sichuan Provincial Health Department of China (110319).

\section{References}

1. Parkin DM, Bray F, Ferlay J and Pisani P: Global cancer statistics, 2002. CA Cancer J Clin 55: 74-108, 2005.

2. Kobayashi H, Mochizuki H, Sugihara K, et al: Characteristics of recurrence and surveillance tools after curative resection for colorectal cancer: a multicenter study. Surgery 141: 67-75, 2007.

3. Bates RC and Mercurio AM: The epithelial-mesenchymal transition (EMT) and colorectal cancer progression. Cancer Biol Ther 4: 365-370, 2005.

4. Brabletz T, Hlubek F, Spaderna S, et al: Invasion and metastasis in colorectal cancer: epithelial-mesenchymal transition, mesenchymal-epithelial transition, stem cells and beta-catenin. Cells Tissues Organs 179: 56-65, 2005.

5. Christiansen JJ and Rajasekaran AK: Reassessing epithelial to mesenchymal transition as a prerequisite for carcinoma invasion and metastasis. Cancer Res 66: 8319-8326, 2006

6. Klymkowsky MW and Savagner P: Epithelial-mesenchymal transition: A cancer researcher's conceptual friend and foe. Am J Pathol 174: 1588-1593, 2009.

7. Spaderna S, Schmalhofer O, Hlubek F, et al: A transient, EMT-linked loss of basement membranes indicates metastasis and poor survival in colorectal cancer. Gastroenterology 131: 830-840, 2006.

8. Eger A, Aigner K, Sonderegger S, et al: DeltaEF1 is a transcriptional repressor of E-cadherin and regulates epithelial plasticity in breast cancer cells. Oncogene 24: 2375-2385, 2005.

9. Ohira T, Gemmill RM, Ferguson K, et al: WNT7a induces E-cadherin in lung cancer cells. Proc Natl Acad Sci U S A 100: 10429-10434, 2003.

10. Chua HL, Bhat-Nakshatri P, Clare SE, et al: NF-kappaB represses E-cadherin expression and enhances epithelial to mesenchymal transit ion of mammary epithelial cells: Potential involvement of ZEB-1 and ZEB-2. Oncogene 26: 711-724, 2007.

11. Graham TR, Zhau HE, Odero-Marah VA, et al: Insulin-like growth factor-I-dependent up-regulation of ZEB1 drives epithelial-to-mesenchymal transition in human prostate cancer cells. Cancer Res 68: 2479-2488, 2008.

12. Spaderna S, Schmalhofer O, Wahlbuhl M, et al: The transcriptional repressor ZEB1 promotes metastasis and loss of cell polarity in cancer. Cancer Res 68: 537-44, 2008.

13. Sánchez-Tilló E, Lázaro A, Torrent R, et al: ZEB1 represses E-cadherin and induces an EMT by recruiting the SWI/SNF chromatin-remodeling protein BRG1. Oncogene 29: 3490-3500, 2010.

14. Xiong H, Hong J, Du W, et al: Roles of STAT3 and ZEB1 proteins in E-cadherin down-regulation and human colorectal cancer epithelial-mesenchymal transition. J Biol Chem 287: 5819-5832, 2012.

15. Waldmann J, Feldmann G, Slater EP, et al: Expression of the zinc-finger transcription factor Snail in adrenocortical carcinoma is associated with decreased survival. Br J Cancer 99: 1900-1907, 2008.

16. Iwatsuki M, Mimori K, Yokobori T, et al: Epithelial-mesenchymal transition in cancer development and its clinical significance. Cancer Sci 101: 293-299, 2010. 
17. Drake JM, Strohbehn G, Bair TB, et al: ZEB1 enhances transendothelial migration and represses the epithelial phenotype of prostate cancer cells. Mol Biol Cell 20: 2207-2217, 2009.

18. Takeyama Y, Sato M, Horio M, et al: Knockdown of ZEB1, a master epithelial-to-mesenchymal transition (EMT) gene, suppresses anchorage-independent cell growth of lung cancer cells. Cancer Lett 296: 216-224, 2010.

19. Singh M, Spoelstra NS, Jean A, et al: ZEB1 expression in type vs type II endometrial cancers: a marker of aggressive disease. Mod Pathol 21: 912-923, 2008.

20. Okugawa Y, Toiyama Y, Tanaka K, et al: Clinical significance of zinc finger E-box binding homeobox 1 (ZEB1) in human gastric cancer. J Surg Oncol 106: 280-285, 2011.

21. Zhou YM, Cao L, Li B, et al: Clinicopathological significance of ZEB1 protein in patients with hepatocellular carcinoma. Ann Surg Oncol 19: 1700-1706, 2012.

22. Shen A, Zhang Y, Yang H, et al: Overexpression of ZEB1 relates to metastasis and invasion in osteosarcoma. J Surg Oncol 105: 830-834, 2012

23. Stridh P, Thessen Hedreul M, Beyeen AD, et al: Fine-mapping resolves Eae23 into two QTLs and implicates ZEB1 as a candidate gene regulating experimental neuroinflammation in rat. PLoS One 5: e12716, 2010.
24. Hidaka T, Nakahata S, Hatakeyama K, et al: Down-regulation of TCF8 is involved in the leukemogenesis of adult T-cell leukemia/ lymphoma. Blood 112: 383-393, 2008.

25. Genetta T, Ruezinsky D and Kadesch T: Displacement of an E-box-binding repressor by basic helix-loop-helix proteins: Implications for B-cell specificity of the immunoglobulin heavychain enhancer. Mol Cell Biol 14: 6153-6163, 1994

26. Fontemaggi G, Gurtner A, Strano S, et al: The transcriptional repressor ZEB regulates $\mathrm{p} 73$ expression at the crossroad between proliferation and differentiation. Mol Cell Biol 21: 8461-8470, 2001.

27. Putzke AP, Ventura AP, Bailey AM, et al: Metastatic progression of prostate cancer and e-cadherin regulation by zeb1 and SRC family kinases. Am J Pathol 179: 400-410, 2011.

28. Argast GM, Krueger JS, Thomson S, et al: Inducible expression of TGFbeta, Snail and Zeb1 recapitulates EMT in vitro and in vivo in a NSCLC model. Clin Exp Metastasis 28: 593-614, 2011.

29. Roy BC, Kohno T, Iwakawa R, et al: Involvement of LKB1 in epithelial-mesenchymal transition (EMT) of human lung cancer cells. Lung Cancer 70: 136-145, 2010.

30. Kim T, Veronese A, Pichiorri F, et al: p53 regulates epithelialmesenchymal transition through microRNAs targeting ZEB1 and ZEB2. J Exp Med 208: 875-883, 2011. 